Check for updates

Cite this: Mater. Adv., 2020 1,1877

Received 18th June 2020,

Accepted 5th August 2020

DOI: 10.1039/d0ma00427h

rsc.li/materials-advances

\title{
Emulating photosynthetic processes with light harvesting synthetic protein (maquette) assemblies on titanium dioxide $\dagger$
}

\author{
Christopher J. Hobbs, ${ }^{a}$ Nicholas Roach, ${ }^{a}$ Pawel Wagner, (D) a Holly van der Salm, ${ }^{b}$ \\ Jonathan E. Barnsley, ${ }^{b}$ Keith C. Gordon, (D) ${ }^{b}$ Goutham Kodali, ${ }^{c}$ \\ Christopher C. Moser, (D) ${ }^{C}$ P. Leslie Dutton, ${ }^{c}$ Klaudia Wagner ${ }^{\star a}$ and \\ David L. Officer (iD *a
}

\begin{abstract}
The emulation of photosynthetic systems has been vigorously pursued for more than thirty years both to assist in the understanding of natural photosynthetic processes as well as for the development of solar-to-fuel systems. While the majority of research has involved the extraction and binding of natural photosynthetic systems to electrodes, there have been limited studies of the electrode immobilisation of synthetic light harvesting proteins. The main focus of this work was to recreate a simplified version of a protein-based, energy generating artificial "photosystem" comprised of an amphiphilic zinc metalloporphyrin (light harvesting unit) ligated to a de novo synthetic protein or maquette. This artificial construct, electrostatically attached to a mesoporous titanium dioxide surface on an FTO electrode, affords the most efficient input (light) - output (photocurrent) protein-based device reported to date. The beneficial effect of the protein can be seen in the improvement of photo voltage through reduction in recombination losses and a more efficient photocurrent generation from a smaller dye loading. This synthetic protein-based construct opens the way for the coupling of this photosystem to catalysts for the development of a whole new generation of biomimetic devices.
\end{abstract}

\section{Introduction}

The replication of photosynthetic processes could not only lay the foundation for sustainable hydrogen production by water splitting but also fuel and food production through carbon dioxide fixation. ${ }^{1,2}$ The key to these photosynthetic outcomes lies in the exquisitely structured photosynthetic charge generation process, in which light is absorbed by a 3-dimensional complex of proteins and light harvesting pigments, and the resulting energy transferred to the "special pair" of bacteriochlorins in the photosynthetic reaction centre (RC). ${ }^{1}$ In order to exploit this finely tuned redox protein architecture, numerous studies into the immobilisation of photosynthetic protein assemblies onto electrodes have been undertaken with

\footnotetext{
${ }^{a}$ ARC Centre of Excellence for Electromaterials Science and the Intelligent Polymer Research Institute, University of Wollongong, NSW 2522, Australia. E-mail:kwagner@uow.edu.au,davido@uow.edu.au

${ }^{b}$ Department of Chemistry, University of Otago, Dunedin 9016, New Zealand

${ }^{c}$ The Johnson Research Foundation and Department of Biochemistry and

Biophysics, University of Pennsylvania, Philadelphia, PA 10104, USA

$\dagger$ Electronic supplementary information (ESI) available: Materials, instrumentation and characterisation, supplementary methods, six supplementary figures and three supplementary tables. See DOI: 10.1039/d0ma00427h
}

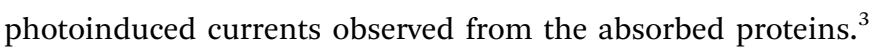
However, the low current densities of these biophotoelectrodes and their instability remain among the challenges for their use in artificial photosynthetic devices. In contrast, there have been few reports of the immobilisation of more robust synthetic light harvesting proteins and these have been limited in scope, with good evidence for interfacial electron transfer but no application into devices, to the best of our knowledge.,

The challenge in building a useful 'artificial photosynthetic' assembly is not in simply mimicking the natural photosynthetic apparatus but utilizing new materials to create and, if possible, simplify or reduce the complexity of the biological system. In 1994, Dutton et al. developed the methodology for the facile production of de novo synthetic protein helices (maquettes), structurally simpler analogs of natural redox proteins. $^{6,7}$ Maquettes are purposely produced to minimise structural complexity of natural proteins, whilst retaining desired function. ${ }^{7,8}$ The maquette structure is typically a four $\alpha$-helix bundle, with each helix 30-40 amino acids in length, containing central histidine residues for ligation. ${ }^{9,10}$ The residues can be used to bind cofactors and develop novel man-made redox proteins. Maquettes have been shown to bind a vast range of cofactors, including flavins, iron-sulfur clusters, 
(a)

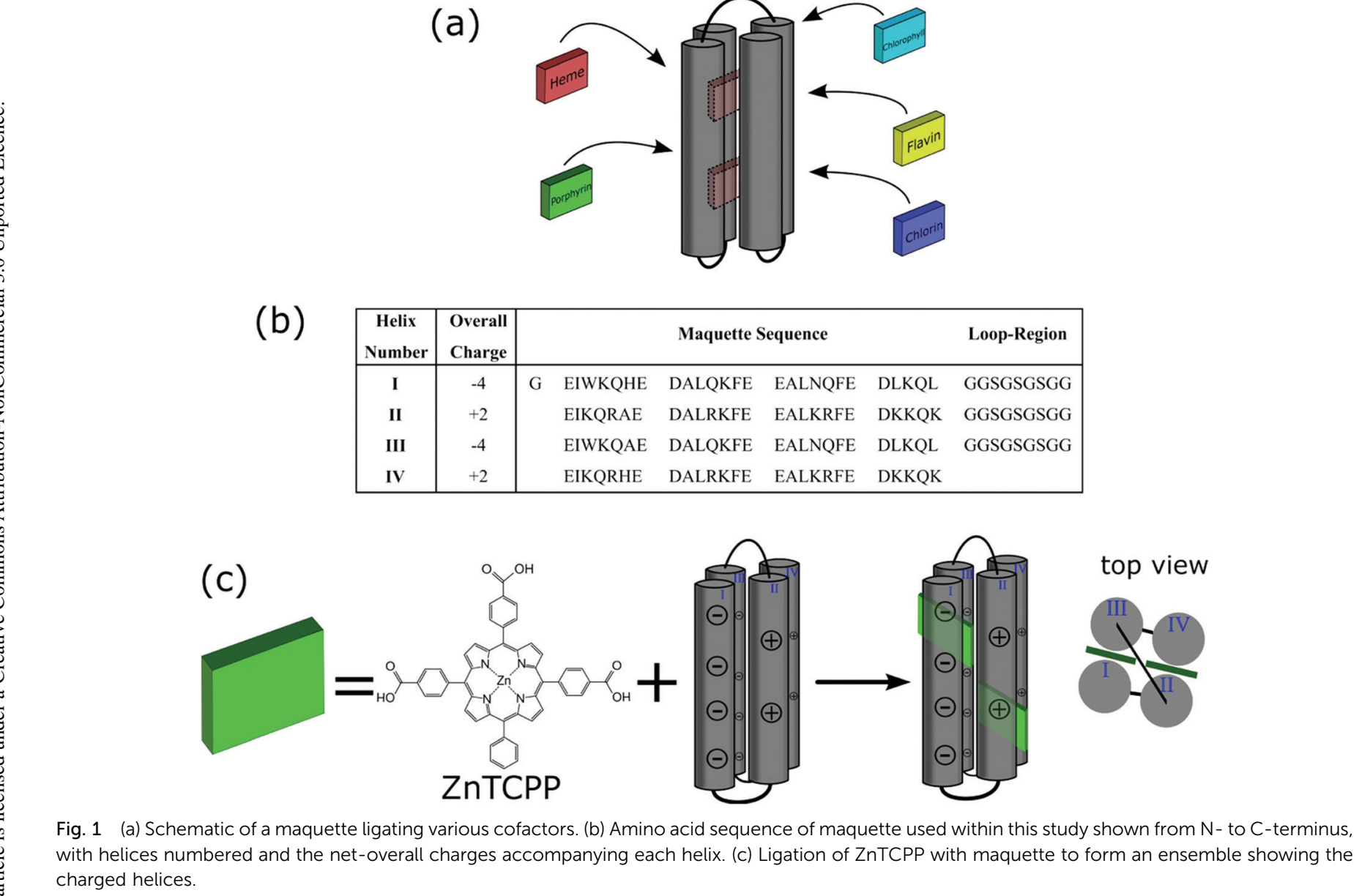

chlorophylls, chlorins, various forms of heme, and porphyrins (Fig. 1a). ${ }^{11-16}$

The incorporation of porphyrins into maquettes offers a unique possibility for photo-induced electron transfer and catalytic properties. It has been demonstrated that not only is a maquette-bound porphyrin more efficiently photo-oxidised than a free porphyrin but also that light-induced electron transfer between the porphyrin complex and an acceptor is faster and higher yielding. ${ }^{15,17}$ Furthermore, by controlling the amino acids and associated charges on the maquette surface, electrostatic interactions with conductive surfaces, and associated electron transfer can be achieved, providing a unique platform on which to build and study a light harvesting reaction centre replica. ${ }^{5,18}$

It is known that lysine residues of peptides can bind to titanium dioxide $\left(\mathrm{TiO}_{2}\right)$ via electrostatic interactions, capable of orienting a peptide on the surface. ${ }^{19,20}$ In this study, a maquette sequence was designed to have appropriate amino acid charges on the maquette surface, in order to interact with $\mathrm{TiO}_{2}$ (Fig. 1b). This presented an opportunity to construct a photoanode to effectively measure photoinduced charge injection into titania by well-established methods. ${ }^{21}$ Here, we demonstrate that a zinc metalloporphyrin strongly binds to the maquette via ligation, and the resulting ensemble can be immobilised onto a
$\mathrm{TiO}_{2}$ electrode. We show that the bound porphyrin maquette ensemble efficiently injects electrons from the photoexcited state of the ligated porphyrin into the conduction band of $\mathrm{TiO}_{2}$, with the maquette inhibiting electron recombination between the conduction band of $\mathrm{TiO}_{2}$ and a redox couple. This work establishes a process for the development of an artificial photosynthetic device utilizing charge separation to effect electron transfer processes.

\section{Results and discussion}

Preparation, purification and characterisation of ZnTCPP-GLmaquette

The zinc metalloporphyrin used for this study is 5,10,15-tri[(4carboxyphenyl)-5-phenylporphyrinato zinc(II)] (ZnTCPP) (Fig. 1c), which was recently shown to ligate strongly in solution to a maquette. ${ }^{22}$ The GL-maquette used comprised 4-alpha helices of heptad repeats, including loop regions of glycine and serine that was based on our previously published maquette sequence with a variation in the amino acid sequences of helices II and IV. ${ }^{22}$ Helices I and III each have an overall charge of -4 , with helices II and IV each containing an overall charge of +2 (Fig. 1b) that can promote electrostatic interactions with the oxide electrode. 
(a)

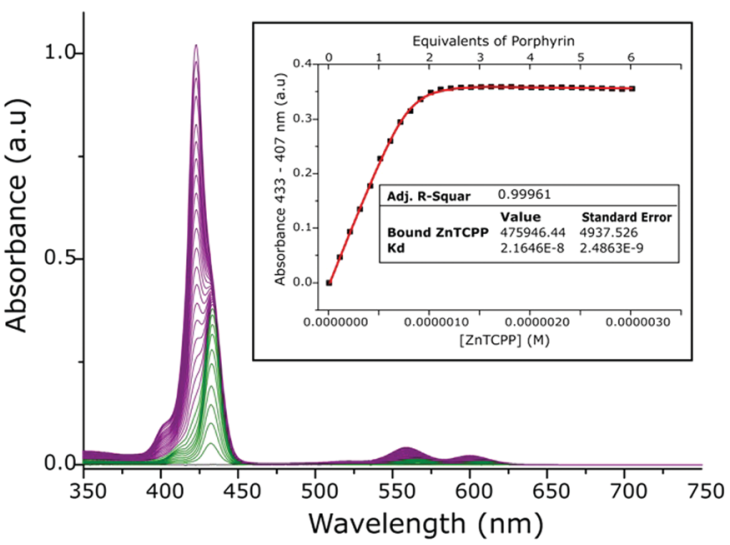

(b)

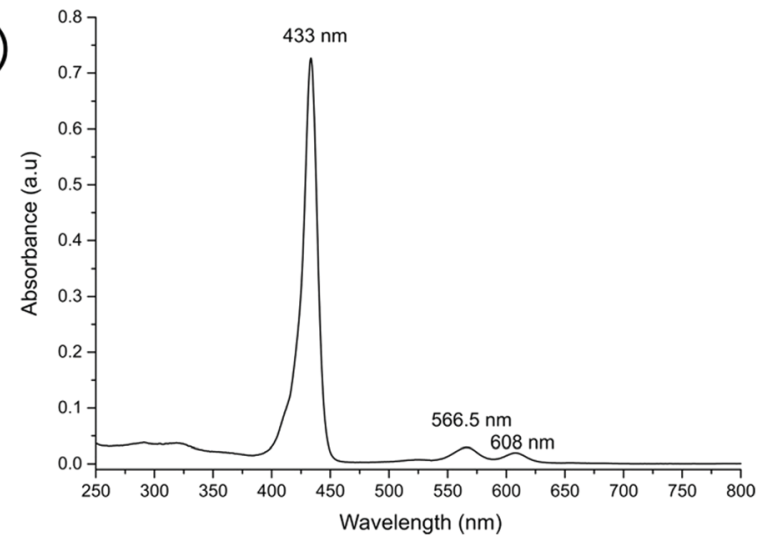

Fig. 2 (a) UV-vis absorption spectra of CHES buffer solution containing maquette with aliquot additions of ZnTCPP. ZnTCPP complexed with GL-maquette (green spectra with maxima at $433 \mathrm{~nm}$ ) and monomeric ZnTCPP in solution (purple spectra with maxima at $422 \mathrm{~nm}$ ) can be observed. A modelled fit of change in absorbance at bound porphyrin maxima compared to concentration of porphyrin present is given in the inset (see Experimental for details). (b) UV-vis spectrum of isolated and concentrated ZnTCPP-GL-maquette ensembles. Absorbance spectrum was obtained from a 100-fold dilution of the concentrated solution resulting from a mixture of 40:100 maquette: ZnTCPP after 5 centrifugal runs in CHES buffer.

The design incorporates two offset histidine residues at the 7 and 112 positions that are each able to ligate to the metallic centre of a single porphyrin.

As previously undertaken for porphyrins binding to maquettes, ${ }^{22}$ the binding of ZnTCPP to the GL-maquette was undertaken in CHES buffer ( $\mathrm{pH}$ 8.5) ensuring solubility of both the maquette and the porphyrin and a binding titration was carried out (see Experimental procedures for details). This showed two distinct species, ligated ZnTCPP with a Soret peak at $433 \mathrm{~nm}$ (green spectrum, Fig. 2a), and unbound ZnTCPP at $422 \mathrm{~nm}$ (purple spectrum, Fig. 2a), becoming apparent after extending the concentration of ZnTCPP beyond the stoichiometric relationship of two ZnTCPPs to one maquette. The distinct red shift of the ZnTCPP absorption spectrum is typical of binding to maquettes, with the ligation of the central zinc to the histidine residue of the maquette influencing the transfer of charge to the porphyrin ring upon complexation. ${ }^{23}$

To determine the strength of binding, a measurement of the dissociation constant along with the extinction coefficient for the ligated ZnTCPP to the maquette was undertaken. The values were obtained by plotting the increase in absorbance at $433 \mathrm{~nm}$ versus the concentration of ZnTCPP, which was then modelled with a fitted curve (inset Fig. 2a). The extinction coefficient of the ligated ZnTCPP $\left(476000 \mathrm{M}^{-1} \mathrm{~cm}^{-1}\right.$ at $\left.433 \mathrm{~nm}\right)$ was comparable to that of ZnTCPP in DMSO $\left(514600 \mathrm{M}^{-1} \mathrm{~cm}^{-1}\right.$ at $429.5 \mathrm{~nm}$, Table S1, ESI $\dagger)$. Dissociation constant $\left(K_{\mathrm{d}}\right)$ values for ZnTCPP ligation were in the nano-molar range $\left(22 \times 10^{-9} \mathrm{M}\right)$, which indicated tight binding, as has previously been shown with maquettes and porphyrins. ${ }^{22}$ In addition, the absorbance versus concentration curve (inset Fig. 2a) shows 2 equivalents of porphyrin present in the GL-maquette, indicating that each histidine ligated a single porphyrin molecule.

Further evidence for binding was provided by resonance Raman spectroscopy (Fig. S1, ESI $\dagger$ ). Metalloporphyrin vibrational modes are known to show considerable sensitivity to the environment, whether in regard to planarity, metal oxidation state/spin or the presence of an auxiliary moiety. ${ }^{24-28}$ Resonance Raman results exhibit spectroscopic changes for ZnTCPP "core size" marker bands with the addition of the GL-maquette (Fig. S1, ESI $\dagger$ ). Relative intensities for bands within $890-1610 \mathrm{~cm}^{-1}$ range increase when compared to the bands at 678 and $714 \mathrm{~cm}^{-1}$. Relative band intensities for $\nu 4$ and $\nu 29\left(1339-1353 \mathrm{~cm}^{-1}\right)$ also change, with $\nu 29$ increasing by over $20 \%$. There is a small red shift of this $\nu 29$ band from 1353 to $1350 \mathrm{~cm}^{-1}$. At the lower energy region, there is a small blue shift of the $1003 \mathrm{~cm}^{-1}$ band $(\nu 15)$ to $1006 \mathrm{~cm}^{-1}$. These spectral changes support the binding of ZnTCPP to the maquette through a histidine residue. It should be noted that Feitelson and Spiro have shown that low frequency (150-250 $\mathrm{cm}^{-1}$ ) resonance Raman data can provide strong evidence for the Zn-histidine stretch, however, no significant bands could be observed in the low frequency regions. ${ }^{29}$

The solution stability of the ZnTCPP-GL-maquette ensemble was also confirmed (Fig. S2, ESI $\dagger$ ) by measuring the absorbance spectrum of the ZnTCPP-GL-maquette over 2 h (Fig. S2a, ESI $\dagger$ ), with the same measurement performed without maquette (Fig. S2b, ESI $\dagger$ ). A stable spectrum under each condition was observed, apparent from the sharp Soret peak and pronounced $\mathrm{Q}$ bands, with no changes occurring over time indicating no release of the ZnTCPP from the maquette.

Isolation and purification of the ZnTCPP-GL-maquette ensembles was undertaken in order to ensure that there was no excess ZnTCPP when binding the ensemble to $\mathrm{TiO}_{2}$. This was performed by subjecting the ZnTCPP-GL-maquette ensemble solution containing an excess of ZnTCPP to multiple centrifuge runs in a protein concentrator tube with a molecular weight cut-off that was less than the molecular weight of the maquette, yet greater than the monomeric porphyrin. The resulting concentrated solution was analysed for purity (Fig. 2b), and for stoichiometric relationship (Fig. S3, ESI $\dagger$ ), affording a maquette to porphyrin ratio of $1: 1.95$. As previously shown, this indicated that the two histidine residues present within the maquette were able to ligate strongly to the monomeric ZnTCPP. The resulting purified solution of ZnTCPP-GL-maquette ensembles was used for immobilisation onto $\mathrm{TiO}_{2}$. 


\section{Immobilisation of $\mathrm{ZnTCPP}-\mathrm{GL}-$ maquette on $\mathrm{TiO}_{2}$}

Immobilisation of the isolated ZnTCPP-GL-maquette ensembles in CHES buffer onto $\mathrm{TiO}_{2}$ was undertaken via electrostatic interaction. At the CHES buffer $\mathrm{pH}(8.5)$, the $\mathrm{TiO}_{2}$ surface is known to be negatively charged $\left(\mathrm{pH}_{\mathrm{IEP}}=5.8-7.5\right),{ }^{30}$ which allowed electrostatic interactions between the helices II and IV of the GL-maquette and $\mathrm{TiO}_{2}$. This dictated the binding geometry as indicated in Fig. 3a, with the ZnTCPP-GL-maquette ensemble lying on the $\mathrm{TiO}_{2}$ with one ligated porphyrin pointing towards the surface and one away from the surface. In order to determine the effect of the protein on the electron transfer from the ligated porphyrins, ZnTCPP, itself, was also immobilised onto $\mathrm{TiO}_{2}$ either from CHES buffer affording electrostatic surface interactions between the carboxylate groups formed in the CHES buffer (Fig. 3b) or directly from an organic solvent leading to covalent surface attachment (Fig. 3c). Covalent attachment to
$\mathrm{TiO}_{2}$ is expected to occur using two binding modes by way of only one or two of the carboxylic groups. ${ }^{31}$

The conditions for binding of the ZnTCPP-GL-maquette ensembles on $\mathrm{TiO}_{2}$ were established by both time and concentration dependent binding studies (Fig. S4 and S5, respectively, ESI $\dagger$ ). These studies established that a maquette concentration of $>20 \mu \mathrm{M}$ and an exposure time of $>24 \mathrm{~h}$ was effective for binding saturation of maquette to the surface of $\mathrm{TiO}_{2}$.

The absorbance spectrum of the ZnTCPP-GL-maquette ensembles on $\mathrm{TiO}_{2}$ was measured to determine whether the ZnTCPP remained ligated within the maquette once immobilised (green line, Fig. 3d). This was compared to the immobilisation of the carboxylate salt of ZnTCPP onto $\mathrm{TiO}_{2}$ from CHES buffer without maquette (red line, Fig. 3d). A distinct sharp Soret peak is observed at $432.5 \mathrm{~nm}$ for the bound ZnTCPP-GLmaquette ensembles, which is identical to the Soret peak for (a)

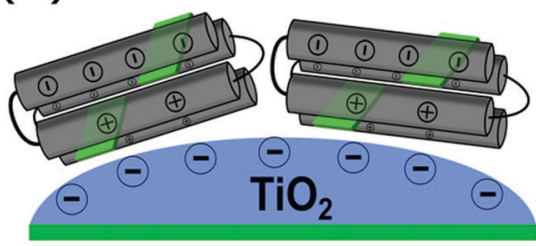

(b)

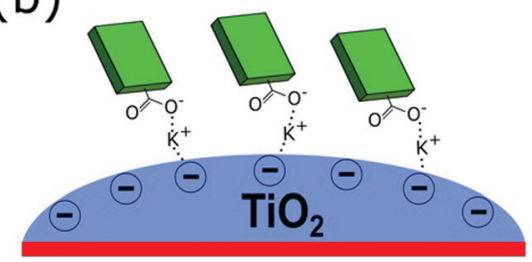

(c)

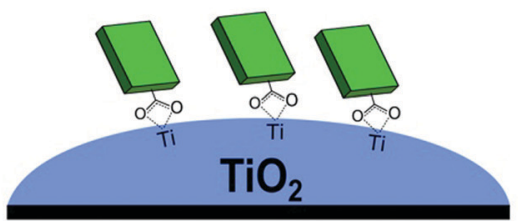

(d)

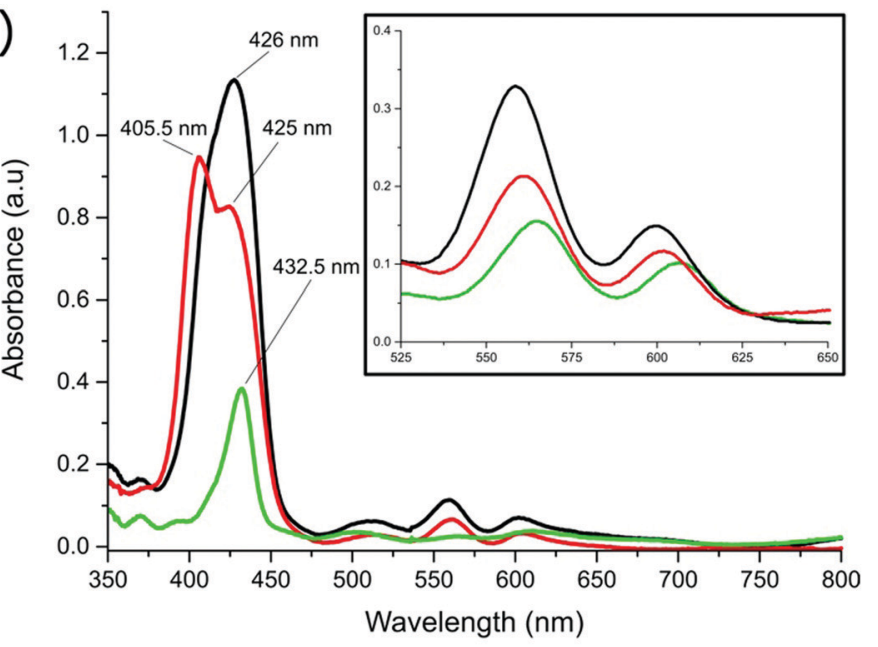

(e)

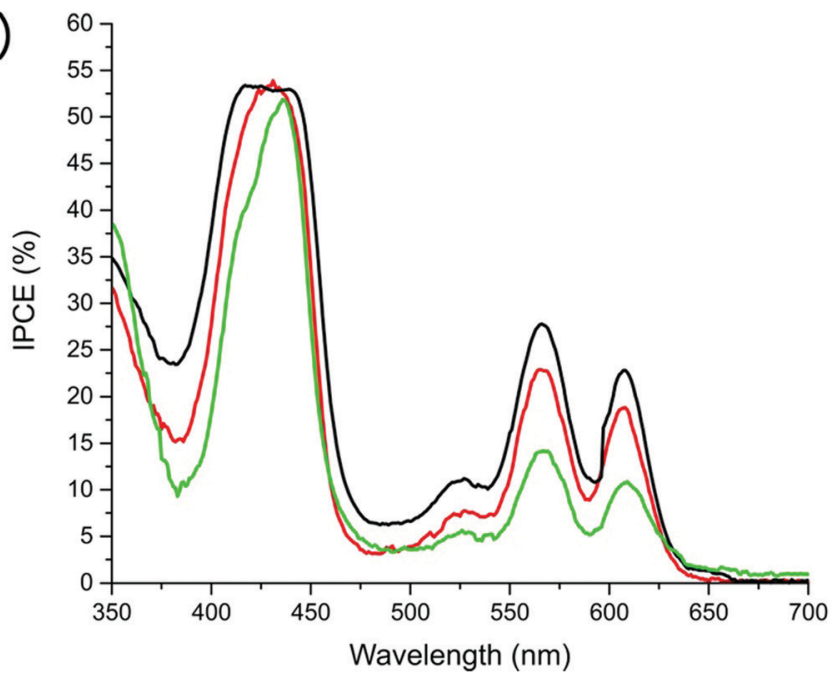

Fig. 3 (a) Illustration of the immobilisation of maquette-ZnTCPP ensembles onto $\mathrm{TiO}_{2}$ from $\mathrm{CHES}$ buffer. (b) Illustration of the binding of the carboxylate salt of $\mathrm{ZnTCPP}$ to $\mathrm{TiO}_{2}$ from CHES buffer solution. (c) Illustration of the covalent binding of $\mathrm{ZnTCPP}$ to TiO ${ }_{2}$ via sensitisation from THF. (d) UV-vis absorption spectra from $0.7 \mu \mathrm{m} \mathrm{TiO}_{2}$ of immobilised GL-maquette-ZnTCPP ensemble from CHES buffer (green line), bound carboxylate salt of ZnTCPP from CHES buffer (red line), and sensitised ZnTCPP from THF (black line). The inset is of modified $2.5 \mu \mathrm{m}$ films of TiO ${ }_{2}$, showing the Q-band regions of the immobilised ZnTCPPs. (e) IPCE spectra from $2.5 \mu \mathrm{m} \mathrm{TiO} 2$ with immobilised GL-maquette-ZnTCPP ensemble from CHES buffer (green line), bound carboxylate salt of ZnTCPP from CHES buffer (red line), and sensitised ZnTCPP from THF (black line). 
Table 1 The photovoltage $\left(V_{\text {oc }}\right)$, photocurrent $\left(J_{s c}\right)$, fill factor $(F F)$, energy conversion efficiency $(\eta)$ and the electron lifetime $(\tau)$ of the solar cells

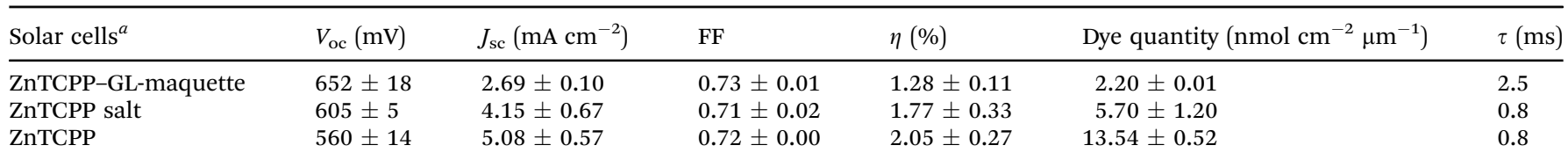

${ }^{a}$ Made up of $2.5 \mu \mathrm{m} \mathrm{TiO}_{2}$ electrodes sensitised with ZnTCPP-GL-maquette, ZnTCPP salt and ZnTCPP using an acetonitrile-based iodide/triiodide redox mediator. The data shown is an average of that from 3 cells \pm standard deviation.

the ZnTCPP-GL-maquette ensembles in solution (Fig. 2b). Furthermore, the Q-band positions (Inset, Fig. 3d and Tables S1 and $\mathrm{S} 2, \mathrm{ESI} \dagger$ ) are also consistent in solution and on $\mathrm{TiO}_{2}$. In addition, the maquette-ligated ZnTCPP bands do not match those of the immobilised ZnTCPP salt (red line, Fig. 3d and Table S2, ESI $\dagger$ ), which are similar to the covalently sensitised ZnTCPP (black line, Fig. 3d and Table S2, ESI $\dagger$ ). It should be noted that the additional peak at $405.5 \mathrm{~nm}$ for the immobilised ZnTCPP salt has been previously reported to arise from the aggregation of porphyrins on oxide surfaces from aqueous solution. ${ }^{32,33}$

\section{Incorporation of ZnTCPP-GL-maquette photoelectrodes into photovoltaic devices}

With the immobilisation of ZnTCPP-GL-maquette ensembles onto a $\mathrm{TiO}_{2}$ electrode achieved, the electrodes could be used for light-driven charge separation within a photovoltaic device such as a dye sensitised solar cell (DSSC). In DSSCs, the energy levels of the photoactive material (dye) need to align with the energy levels of the semiconductor (in order to inject electrons from the photoexcited state) and that of a redox couple, which is required to regenerate the oxidised dye. Electron injection into the conduction band (CB) of the $\mathrm{TiO}_{2}$ semiconductor occurs from the lowest unoccupied molecular orbital (LUMO) of the dye and oxidised dye regeneration from its highest occupied molecular orbital (HOMO). The HOMO energy level can be determined electrochemically from the oxidation potential of the dye attached to the semiconductor while the LUMO energy level can be calculated $\left(E_{\mathrm{LUMO}}=E_{\mathrm{HOMO}}-E_{\text {opt }}\right)$ from the optical band gap $\left(E_{\text {opt }}\right)$, which is determined from the absorption onset at the highest wavelength in the porphyrinmodified $\mathrm{TiO}_{2}$ absorption spectrum (Fig. 3d).

To evaluate the HOMO values, the oxidation potentials of ZnTCPP, ZnTCPP salt bound from CHES buffer, and the ZnTCPP-GL-maquette ensemble were determined using square wave voltammetry (Fig. S6, ESI $\dagger$ ) and found to be $0.91,0.91$ and $0.89 \mathrm{~V} v s$. NHE, respectively. The identical values for the ZnTCPP and ZnTCPP salt bound from CHES buffer indicated that the electronic state of ZnTCPP had not changed despite the difference in binding modes. The significantly lower oxidation current observed for the carboxylate $\mathrm{ZnTCPP}$ on $\mathrm{TiO}_{2}$ (red line, Fig. S6, ESI $\dagger$ ) was due to the aggregative effects hindering electron injection into the electrode (as evident from the absorption spectrum (red line, Fig. 3d)). The lower value for the ZnTCPP-GL-maquette ensemble $(\sim 20 \mathrm{mV})$ is likely due to a change in the electronic state of ZnTCPP as a result of ligation to the maquette through its central zinc. The lower currentresponse for ZnTCPP-GL-maquette compared to the sensitised
ZnTCPP was attributed to the significantly lower concentration of ZnTCPP present in the maquette $(2.30 \pm 0.01$ compared to $13.54 \pm 0.52 \mathrm{nmol} \mathrm{cm}{ }^{-2} \mu \mathrm{m}^{-1}$, respectively, Table 1$)$.

These gave calculated LUMO energy levels of $-1.01,-1.00$ and $-0.93 \mathrm{~V}$ vs. NHE for sensitised ZnTCPP, ZnTCPP salt and the ZnTCPP-GL-maquette ensembles compared to the CB of $\mathrm{TiO}_{2}\left(-0.50 \mathrm{~V} v s\right.$. NHE) ${ }^{21}$ and indicated that it was possible for electrons to be injected into the CB as shown in the energy level diagram in Fig. S7 (ESI $\dagger$ ). In addition, the HOMO values indicated that the dye could be regenerated from the oxidised dye by a redox couple such as $\mathrm{I}^{-} / \mathrm{I}_{3}{ }^{-}$, since the redox potential of the $\mathrm{I}^{-} / \mathrm{I}_{3}{ }^{-}$electrolyte within an organic solvent is $0.35 \mathrm{~V} v s$. NHE and the required driving force for effective regeneration is approximately $0.5 \mathrm{eV} .^{34,35}$

It is noteworthy that while the HOMO-LUMO levels for both the sensitised and ZnTCPP salt were identical, the energy levels for the maquette-bound ZnTCPP differed. This was in agreement with the differences observed in the absorbance spectra, with the electronic state of the maquette-bound ZnTCPP being influenced by ligation. ${ }^{36}$

The influence of the maquette on electron injection from protein-bound zinc porphyrins has been addressed previously. It has been demonstrated that direct photoinduced electron transfer into $\mathrm{TiO}_{2}$ from $\mathrm{Zn}$ protoporphyrins ligated to either cytochrome $C$ or a maquette that are immobilised on $\mathrm{TiO}_{2}$ takes place on the nanosecond time scale,$^{5}$ somewhat slower than for a porphyrin directly attached to $\mathrm{TiO}_{2}$ that is typically subpicosecond. ${ }^{37}$

Sensitised thin film $(2.5 \mu \mathrm{m}) \mathrm{TiO}_{2}$ electrodes were then used for assembly of DSSC constructs, ${ }^{21}$ whose photocurrent density $(J)$ versus voltage $(V)$ curves are given in Fig. S8 (ESI $\dagger$ ) and the full photovoltaic data derived from these curves in Table 1 . The value of the short circuit current density $\left(J_{\mathrm{sc}}\right)$ is a direct measure of the overall light absorption of the dye, efficiency of electron injection of the photoexcited dye and charge collection by the photoanode. If electrons within the photoanode recombine with the redox mediator $\left(\mathrm{I}_{3}{ }^{-}\right)$, this is reflected in a lowering of the open circuit voltage $\left(V_{\mathrm{oc}}\right)$. The fill factor $(\mathrm{FF})$ is a measure of the quality of the photovoltaic device and indicative of the total resistances within the device. The overall DSSC conversion efficiency $(\eta)$ is a combination of these photovoltaic characteristics $\left(\eta=V_{\mathrm{oc}} \times J_{\mathrm{sc}} \times \mathrm{FF}\right)$ and so depends on the balance between the kinetics of electron injection, regeneration, and recombination reactions. ${ }^{38}$ Within porphyrin-based devices, the dye structure and arrangement of dyes on the semiconductor surface both contribute to a lower $V_{\mathrm{oc}}$ than obtained in ruthenium dye-based devices. ${ }^{39,40}$ 
It was evident from the devices utilising the immobilised maquettes containing ligated ZnTCPP that the immobilised ensembles could indeed accomplish light driven electron injection into the $\mathrm{CB}$ of $\mathrm{TiO}_{2}$ as demonstrated by the $\mathrm{mA}$ response in $J_{\text {sc }}(2.69 \pm 0.10 \mathrm{~mA}$, Table 1$)$. As was expected, the two control cells had higher currents, with the sensitised ZnTCPP having the highest measured $J_{\text {sc }}$ and the ZnTCPP salt being slightly lower (5.08 \pm 0.57 and $4.15 \pm 0.67 \mathrm{~mA}$, respectively). However, the quantity of photoactive material (ZnTCPP) present is 6-fold less for devices utilising the ZnTCPP-GL-maquette ensemble compared to those with only ZnTCPP and 2.5-fold less than the ZnTCPP salt devices (Table 1). With $J_{\text {sc }}$ being directly related to the quantity of photoactive material on the semiconducting surface injecting electrons, ${ }^{21}$ these results clearly indicate that when ZnTCPP is ligated to the maquette, the efficiency of the electron injection is higher; there was a higher amount of current than expected coming from the ligated ZnTCPP molecules than the sensitised porphyrins.

The Incident Photon to Current Efficiency (IPCE) was measured to compare the differences in current responses with the absorbance profiles on the $\mathrm{TiO}_{2}$ for the devices (Fig. 3e). The IPCE combines contributions from the light-harvesting efficiency, the efficiency of electron injection from the photoexcited dye into the $\mathrm{TiO}_{2}$ conduction band, and the efficiency of charge collection at the electrodes. In thin $(2.5 \mu \mathrm{m})$ porphyrinsensitised $\mathrm{TiO}_{2}$ DSSCs, the mean diffusion length of electrons is larger than the electron lifetime and, therefore, the charge collection efficiency is assumed to be close to $100 \%{ }^{41}$ It is notable that the total currents extracted from the IPCE curves are comparable to those from the $I-V$ measurements, albeit somewhat smaller due to the lower light intensity used for IPCE measurement; the relative magnitude of the current densities is almost identical. As expected all the IPCE profiles tend to follow the absorbance curves, although it appears that the peak due to the aggregation of the ZnTCPP salt (405.5 nm, red line, Fig. 3d) does not contribute to the photocurrent as might be expected. ${ }^{42}$ Since it has been previously reported that electron injection in porphyrin-based DSSCs mostly occurs from the lowest photoexcited state, ${ }^{43}$ and there is little difference between the IPCEs of these three devices, it can be assumed that injection from the maquette-ligated porphyrin is also mostly from the lowest photoexcited state.

Differences in device performances were further evident in the $V_{\text {oc }}$ (Table 1), where devices with the immobilised ensembles had the highest $V_{\text {oc }}$ compared to the other two devices. Such an increase in $V_{\text {oc }}$ is typically ascribed to a reduction in electron recombination from the $\mathrm{CB}$ of $\mathrm{TiO}_{2}$ to the redox mediator. ${ }^{39,40}$ However, the differences in $V_{\text {oc }}$ and $J_{\text {sc }}$ between devices could also be accounted for by a shift in the $\mathrm{CB}$ edge of $\mathrm{TiO}_{2}$ to higher energy as a result of a $\mathrm{pH}$ increase. ${ }^{44}$ As discussed above for the binding of the maquette, the surface of $\mathrm{TiO}_{2}$ is typically acidic $\left(\mathrm{pH}_{\mathrm{IEP}}=5.8-7.5\right),{ }^{30}$ which would be affected by exposure to the CHES buffer (pH 8.5). Nevertheless, as the ZnTCPP salt and ZnTCPP-GL-maquette ensemble-based devices were both prepared using CHES buffer and show different $V_{\mathrm{oc}} \mathrm{S}$ (Table 1), the increased $V_{\mathrm{oc}}$ of the ensemble devices cannot be solely due to the effect of $\mathrm{pH}$.

Further characterisation through electrochemical impedance spectroscopy (EIS) gives insight into the resistances and capacitance at the various interfaces within the devices. ${ }^{45,46}$ The EIS was carried out on each device at $V_{\text {oc }}$ under 1 sun and the resulting Nyquist and Bode plots are shown in Fig. $4 a$ and b, respectively. To interpret the EIS data, an equivalent circuit model (Fig. 4c) was used to account for the resistances and capacitances associated with each observed interface, and the resulting data is given in Table S3 (ESI $\dagger)$. The series resistance $\left(R_{\mathrm{S}}\right)$ relates to the resistance of the FTO substrates and contact resistances of the circuit, with the series resistance observed to be highly similar between all measured devices (Table S3, ESI $\dagger$ ). The Nyquist plots (Fig. 4a) displayed two significant semicircles for the different electron transfer processes occurring at the various electrochemical interfaces within the devices. ${ }^{46}$ The resistances and capacitances were assigned to each interface observed, where $R_{1}$ and $C_{1}$ corresponds to higher frequencies (smaller semicircle at lower $Z^{\prime}$ value), and $R_{2}$ and $C_{2}$ corresponding to lower frequencies observed (larger semicircle at higher $Z^{\prime}$ value). Typically, diffusion limitations of the redox electrolyte are observed within the lower frequency range. However, due to the short distance between electrodes (25 $\mu \mathrm{m})$, no diffusion impedance can be observed within the investigated frequency range for all devices measured.
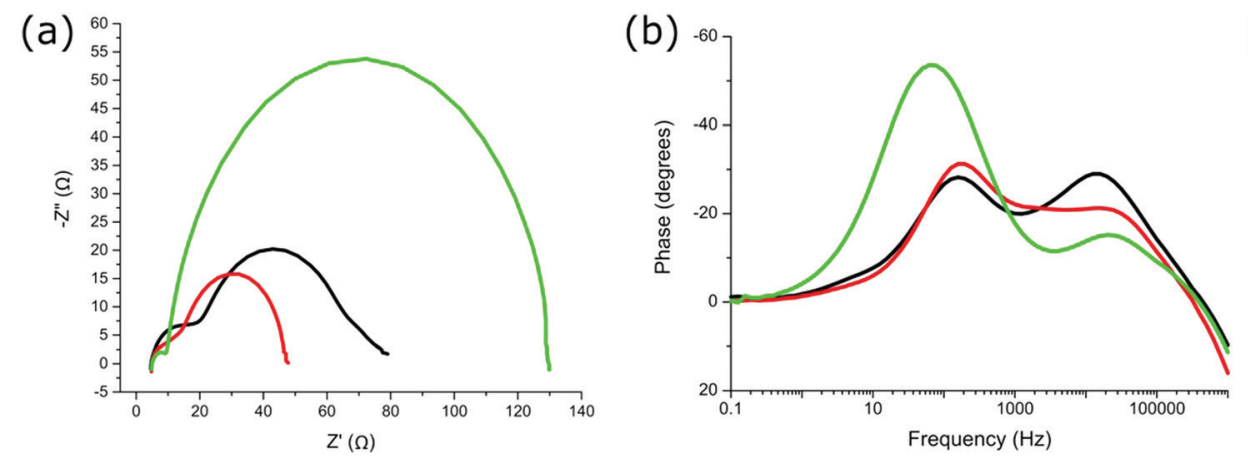

(c)

Fig. 4 (a) Nyquist plot of devices utilizing sensitised ZnTCPP (black line), ZnTCPP salt (red line), and immobilised ZnTCPP-GL-maquette ensemble (green line). (b) Bode plot of devices utilizing sensitised ZnTCPP (black line), ZnTCPP salt (red line), and immobilised ZnTCPP-GL-maquette ensemble (green line). (c) The equivalent circuit diagram used for fitting the EIS data. 
The predominant electron transfer process observed $\left(R_{2}\right.$ and $C_{2}$, larger semicircle) is associated with the reverse electron transfer (recombination) from the conduction band of $\mathrm{TiO}_{2}$ to the redox electrolyte $\left(\mathrm{I}_{3}{ }^{-}\right.$) (Fig. 4a). ${ }^{45}$ The smaller semicircle at the higher frequency range $\left(R_{1}\right.$ and $\left.C_{1}\right)$ is a combination of the charge transfer processes between $\mathrm{TiO}_{2}$ and the FTO glass substrate, between nanoparticles of $\mathrm{TiO}_{2}$, and also between the electrolyte and catalytic platinum layer on the counter electrode. ${ }^{45}$ Since the devices were prepared in an identical manner, the electron transfer between $\mathrm{TiO}_{2}$ and FTO or at the counter electrode was not expected to differ between devices. Therefore, any differences in $R_{1}$ should have come from the transport processes between $\mathrm{TiO}_{2}$ nanoparticles. $R_{1}$ is shown to be highest for devices with sensitised ZnTCPP, with the lowest resistance for devices containing ZnTCPP-GL-maquette ensembles (Table S3, ESI $\dagger$ ). This lower resistance suggests more efficient electron transport at the boundaries of the $\mathrm{TiO}_{2}$ nanoparticles. ${ }^{45} R_{2}$ is the resistance associated with the predominant semicircle (lower frequency range), relating to recombination processes at this $\mathrm{TiO}_{2}$-dye-electrolyte interface. Both $R_{2}$ and $C_{2}$ are notably larger in devices employing ZnTCPP-GL-maquette ensembles (Table S3, ESI $\dagger$ ). The larger capacitance corresponds to a higher density of accessible electronic states at the interface. ${ }^{47}$ The higher resistance indicates that the electron is less likely to recombine from the conduction band of $\mathrm{TiO}_{2}$ back to the redox electrolyte.

One further characteristic obtained from EIS analysis is the electron lifetime associated with the electron recombination from the CB of $\mathrm{TiO}_{2}$ back to the redox mediator. ${ }^{45}$ Electron lifetime is the time of electron survival in the conduction band of $\mathrm{TiO}_{2}$ before recombination with $\mathrm{I}_{3}{ }^{-}$, and is determined by the rate of back reaction to the electrolyte. ${ }^{48}$ Electron lifetimes were calculated from the Bode phase shift versus frequency plots as $\tau \approx 1 / \omega_{\min }=1 / 2 \pi f_{\min }$ where $f_{\min }$ is the frequency at which the phase shift is minimum (Fig. 4b). ${ }^{46}$ The lifetimes for devices with ZnTCPP-GL-maquette ensembles, ZnTCPP salt, and sensitised ZnTCPP were 2.5, 0.8, and $0.8 \mathrm{~ms}$, respectively (Table 1). This result supports the observed $V_{\mathrm{oc}}$ differences shown previously (Table 1), in that a higher electron lifetime is indicative of reduced recombination from the $\mathrm{CB}$ of $\mathrm{TiO}_{2}$ to the electrolyte after photoinduced electron injection and a resulting higher $V_{\mathrm{oc}}$.

It is worthy of note that these results represent a significant advance on previous photoelectrochemical devices fabricated using proteins. The best results to date for natural photosystems immobilised on semiconductor substrates such as $\mathrm{TiO}_{2}$, zinc oxide or hematite incorporated into a photoelectrochemical cell have yielded photocurrents of $100 \mathrm{~s}$ of $\mu \mathrm{A} \mathrm{cm} \mathrm{cm}^{-2}$ and voltages of only up to $0.5 \mathrm{~V}^{3}$ Shah et al. have obtained a $J_{\mathrm{sc}}$ of $4.15 \mathrm{~mA} \mathrm{~cm}^{-2}$ for a biophotoelectrode comprised of photosystem-I on $\mathrm{TiO}_{2}$ but this was in a biased half photoelectrochemical cell configuration using a sacrificial electron donor. ${ }^{49}$ In addition, these proteinbased devices typically degrade over a matter of hours, whereas the maquette photoanodes show no apparent degradation over this time and indeed similar systems have been reported to be stable for weeks. ${ }^{5}$

\section{Conclusions}

In this work, we have demonstrated that a stable complex of an artificial protein and a light harvesting porphyrin can be immobilised onto a $\mathrm{TiO}_{2}$ electrode and that the bound porphyrin protein maquette assembly efficiently injects electrons from the photoexcited state of the ligated porphyrin into the conduction band of $\mathrm{TiO}_{2}$. The beneficial effect of the maquette is demonstrated by the fact that, in the presence of the protein, significantly less porphyrin is required to achieve a similar level of photocurrent as is obtained when the same porphyrin is covalently bound to the $\mathrm{TiO}_{2}$ semiconductor. In addition, there is a substantial increase in photovoltage as a result of the shielding of the $\mathrm{TiO}_{2}$ surface by the protein leading to reduced electron recombination with the redox mediator. While this study has led to the best protein-based photovoltaic device reported, the potential of the porphyrin protein assembly lies in its ability to be coupled to other chemical processes such as occur in photosynthesis. With the design of both the maquette and porphyrin able to be altered to allow the coupling of the electron generation to adjacent catalysts, ${ }^{13}$ this maquetteporphyrin ensemble-based photoanode represents one of the most promising advances for the development of a truly artificial photosynthetic reaction centre.

\section{Experimental}

\section{Porphyrin-maquette binding titration}

The binding titration of porphyrin to maquette was done by adding small aliquots of $1 \mu \mathrm{l}$ from stock porphyrin solution (200 $\mu \mathrm{M}$ porphyrin in DMSO) to $2 \mathrm{ml}$ of $0.5 \mu \mathrm{M}$ maquette solution in CHES buffer (20 mM CHES and $150 \mathrm{mM} \mathrm{KCl}$, adjusted to $\mathrm{pH}=8.5$ with $\mathrm{KOH}$ ), increasing porphyrin concentration by $0.1 \mu \mathrm{M}$ per aliquot. Absorbance spectra were measured after mixing each additional aliquot for $1 \mathrm{~min}$ (Fig. 2a). By plotting the change in absorbance at $433 \mathrm{~nm}$ (absorbance maxima of bound porphyrin) $v s$. the concentration of porphyrin added, the calculated extinction coefficient, dissociation constant $\left(K_{\mathrm{d}}\right)$, and stoichiometric relationship of a modelled fit (Fig. 2a) can be obtained using eqn (1).

$$
\begin{aligned}
& F_{\text {it }}=L_{\text {tot }} \times e_{\text {free }}+\left(e_{\text {bound }}-e_{\text {free }}\right) \\
& \times \frac{\left(K_{\mathrm{d}}+P_{\text {tot }}+L_{\text {tot }}-\left(\left(K_{\mathrm{d}}+P_{\text {tot }}+L_{\text {tot }}\right)^{2}-4 \times P_{\text {tot }} \times L_{\text {tot }}\right)^{0.5}\right)}{2}
\end{aligned}
$$

where $L_{\text {tot }}=$ concentration of added porphyrin $\neq$

$e_{\text {free }}=$ extinction coefficient of free porphyrin $\S$

$e_{\text {bound }}=$ extinction coefficient of bound porphyrin $\S$

$P_{\text {tot }}=$ concentration of maquetteq

$K_{\mathrm{d}}=$ dissociation constant $\S$

\section{$\dashv$ Denotes data parameter.}

$\S$ Denotes free parameter for data fitting.

ๆ Denotes fixed parameter. 


\section{Porphyrin-maquette ensemble in solution}

To obtain a pure and concentrated solution of ZnTCPP-GLmaquette ensembles, $40 \mu \mathrm{M}$ maquette was mixed with $100 \mu \mathrm{M}$ ZnTCPP in $1 \mathrm{ml}$ of CHES Buffer for $1 \mathrm{~h}$, followed by centrifugation in a $3 \mathrm{kDa}$ MWCO (molecular weight cut off) protein concentrator tube (GE Life Sciences). The solution was centrifuged 5 times, with CHES buffer added between spins to a final volume of $1 \mathrm{ml}$. The UV-vis absorbance spectrum of a 100 fold diluted maquette-ZnTCPP ensemble solution was measured to analyse concentration and purity (Fig. 2b).

The concentration was calculated to be $156 \mu \mathrm{M}$ of bound ZnTCPP (based on $\varepsilon=476000 \mathrm{M}^{-1} \mathrm{~cm}^{-1}$ at $433 \mathrm{~nm}$ ). The concentration of maquette was calculated to be $80 \mu \mathrm{M}$, based on the relative change in absorbance at $280 \mathrm{~nm}$, corresponding to the tryptophan absorbance maxima in the maquette (with an extinction coefficient of $11200 \mathrm{M}^{-1} \mathrm{~cm}^{-1}$ ), compared to the change in absorbance at $433 \mathrm{~nm}$ with the increase in ZnTCPP concentration (Fig. S3, ESI $\dagger$ ). The calculated maquette to ZnTCPP ratio was $1: 1.95$.

\section{Binding of porphyrins and ensembles onto $\mathrm{TiO}_{2}$}

Sensitisation of ZnTCPP directly to $\mathrm{TiO}_{2}$ was accomplished by submerging a $\mathrm{TiO}_{2}$ electrode into $200 \mu \mathrm{M}$ ZnTCPP solution in THF for $3 \mathrm{~h}$. Samples were then rinsed in acetonitrile and dried with $\mathrm{N}_{2}$-gas stream before measurements and device fabrication.

Binding of $\mathrm{ZnTCPP}$ to $\mathrm{TiO}_{2}$ from a CHES buffer was accomplished by drop-casting $100 \mu \mathrm{l}$ of $100 \mu \mathrm{M}$ ZnTCPP solution in CHES buffer onto $\mathrm{TiO}_{2}$, with the electrode sealed in a Petri dish (to reduce any evaporation), and left for $48 \mathrm{~h}$ in the dark at $4{ }^{\circ} \mathrm{C}$. The treated electrode was then rinsed in CHES buffer and dried with $\mathrm{N}_{2}$-gas stream before measurements and device fabrication.

The isolated ZnTCPP-GL-maquette ensemble solution (undiluted) with known concentrations of bound ZnTCPP and maquette (Fig. S3, ESI $\dagger$ ) was diluted to give $100 \mu \mathrm{M}$ ligated ZnTCPP (and hence $51 \mu \mathrm{M}$ maquette) in CHES buffer. The resulting solution was drop-casted onto $\mathrm{TiO}_{2}$, with the electrode sealed in a Petri dish, left for $48 \mathrm{~h}$ in the dark at $4{ }^{\circ} \mathrm{C}$. The treated electrodes were then rinsed in CHES buffer and dried with $\mathrm{N}_{2}$-gas stream before measurements and device fabrication.

\section{Device assembly}

$\mathrm{TiO}_{2}$ films were prepared on FTO substrates (Hartford TEC8, $2.3 \mathrm{~mm}, R_{\mathrm{S}}<8 \Omega \square^{-1}$ ) by screen-printing $4 \times 4 \mathrm{~mm}$ areas of one $2.5 \mu \mathrm{m}$ transparent layer (DSL 18 NR-T paste, Dyesol), or one 2-fold terpineol diluted (mass to mass) layer $(0.7 \mu \mathrm{m}) \mathrm{using}$ a $96 \mathrm{~T}$ screen. All film thicknesses were measured using a Dektak 150 Profilometer. The $\mathrm{TiO}_{2}$ films were sintered stepwise using a programmable hot plate with a maximum temperature of $500{ }^{\circ} \mathrm{C}$. Each film was reheated to $450{ }^{\circ} \mathrm{C}$ for $20 \mathrm{~min}$ and cooled to $110{ }^{\circ} \mathrm{C}$ for $30 \mathrm{~min}$ before any treatment.

Counter electrodes were prepared by platinising TEC8 FTO with an ethanolic $10 \mathrm{mM} \mathrm{H}_{2} \mathrm{PtCl}_{6}$ solution at $400{ }^{\circ} \mathrm{C}$ for $20 \mathrm{~min}$.

Sandwich-type DSSCs were assembled using a $25 \mu \mathrm{m}$ Hymilan sealant. An electrolyte solution composed of $0.6 \mathrm{M}$ 1-butyl-3methylimidazolium iodide (BMII), 0.5 M 4-tert-butylpyridine,
$0.1 \mathrm{M} \mathrm{LiI}$, and $0.03 \mathrm{M} \mathrm{I}_{2}$ in a solvent mixture of $85: 15$ acetonitrile/ valeronitrile was injected between the electrodes through a hole in the counter electrode, which was subsequently sealed. Devices were measured under 1 sun irradiation.

\section{Conflicts of interest}

There are no conflicts to declare.

\section{Acknowledgements}

This work was supported by Australian Research Council (ARC) Discovery Project DP 150104532 and the ARC Centre of Excellence Scheme (Project Number CE 140100012). The authors thank the Australian National Fabrication Facility for facilities. The support of the New Zealand MacDiarmid Institute for Advanced Materials and Nanotechnology is gratefully acknowledged. This research was carried out as part of the Photosynthetic Antenna Research Center (PARC), an Energy Frontier Research Center funded by the U.S. Department of Energy, Office of Science, Office of Basic Energy Sciences, under Award DESC0001035 supporting G. K., P. L. D. and C. C. M. in maquette construction and characterisation.

\section{Notes and references}

1 J. Barber, Chem. Soc. Rev., 2009, 38, 185-196.

2 C. Koroneos, A. Dompres, G. Roumbus and N. Moussiopoulos, Int. J. Hydrogen Energy, 2004, 29, 1443-1450.

3 V. M. Friebe and R. N. Frese, Curr. Opin. Electrochem., 2017, 5, 126-134.

4 H. C. Fry, Y. Liu, N. M. Dimitrijevic and T. Rajh, Nat. Commun., 2014, 5, 4606.

5 E. Topoglidis, B. Discher, C. C. Moser, P. L. Dutton and J. Durrant, ChemBioChem, 2003, 4, 1332-1339.

6 B. M. Discher, D. Noy, J. Strzalka, S. Ye, C. C. Moser, J. D. Lear, J. K. Blasie and P. L. Dutton, Biochemistry, 2005, 44, 12329-12343.

7 D. Robertson, R. Farid, C. C. Moser, J. Urnauer, S. Mulholland, R. Pidikiti, J. Lear, J. Wand, W. Degrado and P. L. Dutton, Nature, 1994, 368, 425-432.

8 J. Bryson, S. Betz, H. Lu, D. Suich, H. Zhou, K. O'Neil and W. Degrado, Science, 1995, 270, 935-941.

9 P. L. Dutton and C. C. Moser, Faraday Discuss., 2011, 148, 443-448.

10 B. Gibney, F. Rabanal, J. Skalicky, J. Wand and P. L. Dutton, J. Am. Chem. Soc., 1997, 119, 2323-2324.

11 R. E. Sharp, C. C. Moser, F. Rabanal and P. L. Dutton, Proc. Natl. Acad. Sci. U. S. A., 1998, 95, 10465-10470.

12 S. Mulholland, B. Gibney, F. Rabanal and P. L. Dutton, Biochemistry, 1999, 38, 10442-10448.

13 I. Willner, V. Heleg-Shabtai, E. Katz, H. K. Rau and W. Haehnel, J. Am. Chem. Soc., 1999, 121, 6455-6468.

14 L. Eggink and J. K. Hoober, J. Biol. Chem., 2000, 275, 9087-9090. 15 A. R. Razeghifard and T. Wydrzynski, Biochemistry, 2003, 42, 1024-1030. 
16 S. Huang, R. Koder, M. Lewis, J. Wand and P. L. Dutton, Proc. Natl. Acad. Sci. U. S. A., 2004, 101, 5536-5541.

17 V. Tipmanee, H. Oberhofer, M. Park, K. S. Kim and J. Blumberger, J. Am. Chem. Soc., 2010, 132, 17032-17040.

18 X. Chen, B. Discher, D. Pilloud, B. Gibney, C. C. Moser and P. L. Dutton, J. Phys. Chem., 2002, 103, 617-624.

19 A. D. Roddick-Lanzilotta and A. J. McQuillan, J. Colloid Interface Sci., 1999, 217, 194-202.

20 M. Kato, T. Cardona, A. W. Rutherford and E. Reisner, J. Am. Chem. Soc., 2013, 135, 10610-10613.

21 A. Hagfeldt, G. Boschloo, L. Sun, L. Kloo and H. Pettersson, Chem. Rev., 2010, 110, 6595-6663.

22 G. Kodali, J. A. Mancini, L. A. Solomon, T. V. Episova, N. Roach, C. J. Hobbs, P. Wagner, O. A. Mass, K. Aravindu, J. E. Barnsley, K. C. Gordon, D. L. Officer, P. L. Dutton and C. C. Moser, Chem. Sci., 2017, 8, 316-324.

23 M. Nappa and J. S. Valentine, J. Am. Chem. Soc., 1978, 100, 5075-5080.

24 J. A. Shelnutt, K. Alston, J. Y. Ho, N. T. Yu, T. Yamamoto and J. M. Rifkind, Biochemistry, 1986, 25, 620-627.

25 J. A. Shelnutt, C. J. Medforth, M. D. Berber, K. M. Barkigia and K. M. Smith, J. Am. Chem. Soc., 1991, 113, 4077-4087.

26 T. G. Spiro, J. D. Stong and P. Stein, J. Am. Chem. Soc., 1979, 101, 2648-2655.

27 T. G. Spiro and T. C. Strekas, J. Am. Chem. Soc., 1974, 96, 338-345.

28 Y.-H. Zhang, W. Zhao, P. Jiang, L.-J. Zhang, T. Zhang and J. Wang, Spectrochim. Acta, Part A, 2010, 75, 880-890.

29 J. Feitelson and T. G. Spiro, Inorg. Chem., 1986, 25, 861-865.

30 R. Beranek, Adv. Phys. Chem., 2011, 1-20.

31 L. Zhang and J. M. Cole, ACS Appl. Mater. Interfaces, 2015, 7, 3427-3455.

32 R. Azumi, M. Matsumoto, Y. Kawabata, S. Kuroda, M. Sugi, L. G. King and M. J. Crossley, J. Phys. Chem., 1993, 97, 12862-12869.

33 G. A. Schick, I. C. Schreiman, R. W. Wagner, J. S. Lindsey and D. F. Bocian, J. Am. Chem. Soc., 1989, 111, 1344-1350.
34 G. Boschloo and A. Hagfeldt, Acc. Chem. Res., 2009, 42, 1819-1826.

35 I. Montanari, J. Nelson and J. R. Durrant, J. Phys. Chem. B, 2002, 106, 12203-12210.

36 C.-l. Lin, M.-Y. Fang and S.-H. Cheng, J. Electroanal. Chem., 2002, 531, 155-162.

37 A. S. Hart, C. B. Kc, H. B. Gobeze, L. R. Sequeira and F. D'Souza, ACS Appl. Mater. Interfaces, 2013, 5, 5314-5323.

38 S. A. Haque, E. Palomares, B. M. Cho, A. N. M. Green, N. Hirata, D. R. Klug and J. R. Durrant, J. Am. Chem. Soc., 2005, 127, 3456-3462.

39 M. J. Griffith, M. James, G. Triani, P. Wagner, G. G. Wallace and D. L. Officer, Langmuir, 2011, 27, 12944-12950.

40 M. J. Griffith and A. J. Mozer, in Solar Cells, ed. L. A. Kosyachenko, INTECH Open Access Publisher, Rijeka, 2011, ch. 16, vol. 2 .

41 A. J. Mozer, P. Wagner, D. L. Officer, G. G. Wallace, W. M. Campbell, M. Miyashita, K. Sunahara and S. Mori, Chem. Commun., 2008, 4741-4743.

42 J. L. McHale, J. Phys. Chem. Lett., 2012, 3, 587-597.

43 K. Wagner, M. J. Griffith, M. James, A. J. Mozer, P. Wagner, G. Triani, D. L. Officer and G. G. Wallace, J. Phys. Chem. C, 2011, 115, 317-326.

44 G. Rothenberger, D. Fitzmaurice and M. Graetzel, J. Phys. Chem., 1992, 96, 5983-5986.

45 T. Hoshikawa, M. Yamanda, R. Kikuchi and K. Eguchi, J. Electrochem. Soc., 2005, 152, E68-E73.

46 R. Kern, R. Sastrawan, J. Ferber, R. Stangl and J. Luther, Electrochim. Acta, 2002, 47, 4213-4225.

47 F. Fabregat-Santiago, G. Garcia-Belmonte, I. Mora-Sero and J. Bisquert, Phys. Chem. Chem. Phys., 2011, 13, 9083-9118.

48 J. Bisquert, F. Fabregat-Santiago, I. Mora-Seró, G. GarciaBelmonte and S. Giménez, J. Phys. Chem. C, 2009, 113, 17278-17290.

49 V. B. Shah, W. R. Henson, T. S. Chadha, G. Lakin, H. Liu, R. E. Blankenship and P. Biswas, Langmuir, 2015, 31, 1675-1682. 